\title{
ON THE HECUBA GAP
}

\author{
S.FERRAZ-MELLO \\ Instituto Astronômico e Geofísico, Universidade de São Paulo, \\ Caixa Postal 9638, 01065-São Paulo, SP, Brasil. \\ sylvio@vax.iagusp.usp.br
}

\begin{abstract}
An asteroid captured in the Hecuba gap (2/1 resonance with Jupiter) may remain there for a long time before escaping. However, the study of the diffusion of orbits in the gap indicates an escape timescale in the range $10^{7}-10^{9}$ years. The short-period perturbations of Jupiter's orbit play a determinant role in the creation of the stochasticity responsible for the escape.
\end{abstract}

\section{The Hecuba gap}

The Hecuba gap is the minimum in the asteroid distribution located at the place where the asteroids have a mean-motion resonance with Jupiter in the ratio 2:1. An asteroid captured in this resonance may remain there for a long time before escaping. However, the study of the diffusion of orbits in the gap, indicates an escape timescale of the order of $10^{8}$ years or, more precisely, in the range $10^{7}-10^{9}$ years, providing evidences linking the Hecuba gap with the global stochasticity of the 2/1 asteroidal resonance (Ferraz-Mello, 1994a,b; Franklin, 1994; Ferraz-Mello et al., 1995) This scenario contradicts previous ideas in several respects. First of all, it is well known that the inner chaotic region of the $2 / 1$ resonance, in the planar elliptic restricted three-body problem, is shielded from the outside by an extended bunch of regular motions (see the Poincaré maps in FerrazMello 1994a,b). It is also known that, when the long-period perturbations of Jupiter's orbit are taken into account, these regular barriers are not destroyed (Morbidelli and Moons, 1993). The assumptions extending these facts to the exact model led to accept the impossibility of a chaotic diffusion able to drive these orbits off this region in a time of the order of the 
age of the asteroid belt, making necessary ad-hoc cosmogonic hypotheses to explain the almost absence of asteroids in the gap. But all Lyapunov times computed in the resonant region with an exact Sun-Jupiter-Saturn-asteroid model (Ferraz-Mello, 1994a; Franklin, 1994) point to a global stochasticity whose origin may be searched in the Chirikov regime created by the complex overlap of low- and high-order secondary resonances.

The most serious criticisms to this scenario cames from the scarcity of precise simulations actually showing the diffusion. In fact, only a few results of numerical integrations of fictitious asteroids in the $2 / 1$ resonance are available. They are 5 early numerical integrations reported by Wisdom (1987), one by Scholl (see fig. 7 in Ferraz- Mello, 1994b) and some more recent ones obtained by Henrard et al.(1995). Notwithstanding the limited timespan of these integrations (the longest reaching only $12 \mathrm{Myr}$ ), half of them show important diffusing orbital processes, and the later one shows a possible path, through moderate inclinations, leading from the inner chaotic region $(e \sim 0.1)$ to high eccentricities. Some more long runs were done by Franklin (1994) in the frame of a planar model. In these runs most of the solutions escaped, but three solutions remained in the resonance zone for more than 120 Myr.

The aim of this communication is to present some results concerning the stochasticity of the $2 / 1$ resonance obtained by means of a planar symplectic mapping and show that the failure of the existing averaged models in showing a stronger chaotic behaviour cames from the fact that the long-period perturbations of Jupiter's orbit are not able, alone, to produce such chaos in a planar model. The situation changes drastically when we add, to the model, the short-period perturbations whose arguments are $\cos \left(2 \lambda_{\text {Jup }}-5 \lambda_{\text {Sat }}\right)$ and $\cos \left(\lambda_{\text {Jup }}-2 \lambda_{\text {Sat }}\right)$.

\section{The mapping}

The mapping used is a modified form of the symplectic mapping introduced by Hadjidemetriou $(1988,1991)$. The equations of this mapping are those of the canonical transformation spanned by the Jacobian generator $W=$ $\mathcal{I}+\tau H$ where $H$ is the given averaged Hamiltonian, $\mathcal{I}$ the generator of the identical transformation and $\tau$ is the map step. The transformation is such that the mapping has the same fixed points - with the same stability characteristics - as the surface of section of the averaged system.

The original Hadjidemetriou formulation of the mapping was modified. The classical Laplacian expansion of the averaged potential of the disturbing forces, problematic in high eccentricities, was substituted by the asymmetric expansion of Ferraz-Mello and Sato (1989) which gives a good representation of it even in high eccentricities provided that the motion is 
TABLE 1. Main long- and short-period oscillations in $e_{1} \cdot e^{i \varpi_{1}}$

\begin{tabular}{rrrrr}
\hline Term & & Amplitude & Frequency & \\
\hline$g_{5}$ & + & 0.0441872 & 4.257493 & $\operatorname{arcsec} / \mathrm{yr}$ \\
$g_{6}$ & - & 157002 & 28.245530 & \\
$-g_{5}+2 g_{6}$ & - & 5735 & 52.233567 & \\
$2 g_{5}-g_{6}$ & + & 142 & -19.730544 & \\
$g_{7}$ & + & 18139 & 3.086756 & \\
$-g_{5}+g_{6}+g_{7}$ & + & 1982 & 27.074793 & \\
$g_{5}+g_{6}-g_{7}$ & + & 1936 & 29.416267 & \\
\hline$-\lambda_{1}+2 \lambda_{2}+\varpi_{1}$ & & 0.000646 & $\dot{\varpi}_{1}-21264.4$ & $\operatorname{arcsec} / \mathrm{yr}$ \\
$-2 \lambda_{1}+5 \lambda_{2}+\varpi_{1}$ & & 364 & $\dot{\varpi}_{1}+1467.2$ & \\
\hline
\end{tabular}

a libration of moderate amplitude.

We consider, generically, an asteroid in a resonance $(p+q): p$ with Jupiter and moving in the same plane as the planet. We introduce the long-period angular variables:

$$
\psi=\frac{p+q}{q} \lambda_{1}+\frac{p}{q} \lambda, \quad \sigma=\psi-\varpi
$$

and their canonical conjugate actions, respectively $P$ and $J$. The basic function of Hadjidemetriou's mapping is the Jacobian generator

$W\left(J_{n+1}, P_{n+1}, \sigma_{n}, \psi_{n}, t_{n}\right)=\sigma_{n} J_{n+1}+\psi_{n} P_{n+1}+\tau \mathcal{H}\left(J_{n+1}, P_{n+1}, \sigma_{n}, \psi_{n}, t_{n}\right)$

where $\tau$ is the map step and $H$ is the averaged Hamiltonian

$$
\mathcal{H}=-\frac{\mu^{2}}{2 L^{2}}-L \frac{p+q}{q} n_{1}-R
$$

$L=\sqrt{\mu a}=-(J+P), \mu$ is the square of the Gaussian constant, $n_{1}$ is the mean motion of Jupiter and $R$ is the disturbing potential averaged over the synodic period. All results in this paper were obtained taking the step $\tau$ equal to the initial synodic period of the asteroid with respect to Jupiter.

When the orbit of Jupiter is kept fixed, the only possible secular resonance is associated with the angle $\varpi-\varpi_{1}$ (with $\varpi_{1}=$ const. ). This secular resonance is responsible for the corotation zone seen in the middle of the resonance, at high eccentricities (see Ferraz-Mello, 1994a). When the actual perihelion of Jupiter is introduced, the corotation zone remains almost the same since its motion is very slow. However, the resulting increase in the number of degrees of freedom may give rise to chaotic regions which 
were not apparent in the restricted model (Morbidelli and Moons, 1993). Table I gives the amplitudes of the long- and short-period perturbations in the complex quantity $e_{1} . e^{i \varpi_{1}}$ used in this investigation, according with, respectively, Nobili et al.(1989) and Simon and Bretagnon (1975). In this table $g_{5}, g_{6}$ and $g_{7}$ represent the proper perihelion of Jupiter, Saturn and Neptune, respectively; $\lambda_{2}$ is the mean longitude of Saturn. For the sake of allowing a direct comparison of the long- and short-period perturbations of Jupiter's perihelion, the perturbation equations of the eccentricity and perihelion given by Simon and Bretagnon (1975), which are actually used in the mapping, were slightly modified.

We consider, also, the most important perturbations in the mean longitude of Jupiter, corresponding to the near commensurability $5: 2$ of its mean-motion with Saturn's According with Simon and Bretagnon (1975), these perturbation are given by

$$
\delta \lambda_{1}=-5.164 \times 10^{-4} \cos \left(2 \lambda_{1}-5 \lambda_{2}\right)-51.502 \times 10^{-4} \sin \left(2 \lambda_{1}-5 \lambda_{2}\right) .
$$

\section{Scaled models}

In order to go over the barriers that a slow chaos puts to investigation, we may introduce artificial parameter variations able to accelerate that mechanisms. We have used scaled models related to the real problem by a mass gauge. In the case of the restricted model, it is simply a factor multiplying the mass of Jupiter. The main frequencies of the planar model - the frequency of the perihelion motion and the libration frequency - are expected to be roughly multiplied, respectively, by this gauge and by its square root. The structure of the resonance web will be altered. Thus, in order to keep this alteration small and avoid new secondary resonances to appear and create artificial domains with different diffusion patterns, we limit ourselves to gauges close to 1 .

In what concerns the motion of Jupiter, the frequencies of the longperiod perturbations are amplified in the same proportion while the amplitudes remain the same. A mass gauge is likely expected to amplify only the amplitudes of the short-period perturbation of Jupiter's orbit; however, in order to preserve the resonance web, as much as possible, also the frequencies are linearly amplified in the scaled model.

One must kept in mind that no perfect scaling is possible in a non-linear problem. Therefore, results got with a scaled model cannot be considered as true before verified with non-scaled models. This rule is followed in this investigation. 

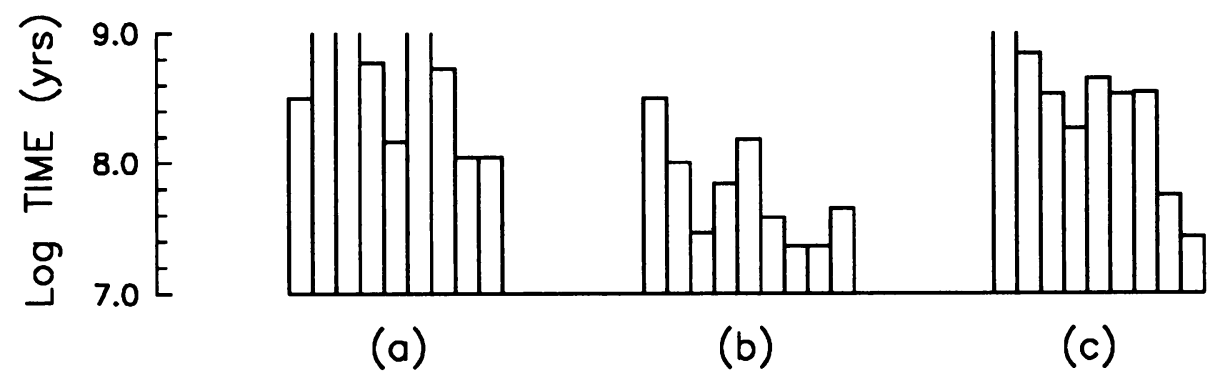

Figure 1. Median times to reach $e=0.56$ in three cases: (a) Jupiter orbit with short-period perturbations only; scaled $M_{g}=1.4$. (b) Jupiter orbit with short- and long-period perturbations; scaled $M_{g}=1.4$. (c) Jupiter orbit with short- and long-period perturbations; non scaled $\left(M_{g}=1.0\right)$. In each histogram the initial eccentricity varies from 0.11 to 0.35 , from left to right, with a 0.03 step.

TABLE 2. Solutions reaching the limit value $e=0.56\left(M_{g}=1.4\right)$

\begin{tabular}{lcc}
\hline $\begin{array}{l}\text { Perturbations in } \\
\text { Jupiter's Motion }\end{array}$ & $\begin{array}{c}\text { Limit reached } \\
\text { in less than } 10^{9} \text { yrs }\end{array}$ & $\begin{array}{c}\text { Limit reached } \\
\text { in less than } 10^{8} \text { yrs }\end{array}$ \\
\hline Long-period only & $15 \%$ & $10 \%$ \\
Short-period only & $68 \%$ & $32 \%$ \\
both & $95 \%$ & $72 \%$ \\
\hline
\end{tabular}

\section{Results and Conclusions}

Thanks to the speed of the mapping, we have been able to made a great deal of different experiments. A more systematic study was done selecting a grid of initial conditions delimited by initial eccentricities in the interval $0.11-$ 0.35 and semi-major axes in the interval 3.272-3.284 AU (the middle of the resonance lies close to $3.276 \mathrm{AU}$ ). Initial values of $\sigma$ and $\varpi-\varpi_{1}$ were fixed at $0^{\circ}$. Scaled models were computed in a net of 40 points in this domain with many different combinations of the perturbations included in Jupiter's orbit. Table II summarizes results obtained using a mass gauge 1.4 (that is, the mass of Jupiter and the frequencies of the perturbations introduced in Jupiter's orbit were multiplied by 1.4) and including all perturbations of table 1.

Table 2 shows that, when the model adopted for Jupiter's orbit include both short- and long-period perturbations, the transition allowing its eccentricity to increase happened in $95 \%$ of the studied cases. The limit $e=0.56$ was set arbitrarily (it corresponds to a perihelion distance $1.44 \mathrm{AU}$ ). The results show clearly that, in the long run, in the planar reduction of the 
$2 / 1$ resonance, the perturbations of Jupiter's orbit whose frequencies are $5 n_{2}-2 n_{1}$ and $2 n_{2}-n_{1}$ are more important than the long-period perturbations of Jupiter's orbit. The distribution of the times necessary to reach the limit $e=0.56$ as a function of the initial eccentricity, from 0.11 to 0.35 in steps of 0.03, are shown in Fig. 1 (a) and (b).

The important role played by the short-period perturbations of Jupiter's orbit are confirmed when the experiments are done without any scaling (mass gauge $M_{g}=1.0$ ). When both short- and long-period perturbations of Jupiter's orbit are considered, $38 \%$ of the solutions reached $e=0.56$ in less than $10^{8}$ years and $87 \%$ reached it in less than $10^{9}$ years. The distribution of the times necessary to reach the limit $e=0.56$ as a function of the initial eccentricity, from 0.11 to 0.35 in steps of 0.03 , is shown in Fig. 1(c).

These results show that the global stochasticity verified with numerical 4-body models, including Saturn, does not came from the direct action of Saturn, as it was sometimes suspected, but from the main short-period perturbations of Jupiter's orbit.

\section{References}

Ferraz-Mello, S.: 1994a, "The convergence domain of the Laplacian expansion of the disturbing function". Celest. Mech. Dyn. Astron. 58, 37-52.

Ferraz-Mello, S.: 1994b, "Dynamics of the 2:1 asteroidal resonance". Astron. J. 108, 2330-2337.

Ferraz-Mello, S., Dvorak. R. \& Michtchenko, T.A. (1994). "Chaos and the depletion of asteroids in resonant orbits", In From Newton to Chaos, (A.E.Roy and B.Steves, eds.) Plenum Press, New York, pp. 157-169

Ferraz-Mello, S. and Sato, M. 1989, "A very-high-eccentricity asymmetric expansion of the disturbing function near resonances of any order". Astron. Astrophys. 225, 541547.

Franklin, F. (1994). "An examination of the relation between chaotic orbits and the Kirkwood gap at the 2:1 resonance" Astron. J. 107, 1890.

Hadjidemetriou, J.D.: 1988, "Algebraic mappings near a resonance with an application to asteroid motion". In Long-Term Dynamical Behaviour of Natural and Artificial $N$-Body Systems (A.E.Roy, ed.), Kluwer, Dordrecht, 257-276.

Hadjidemetriou, J.D.: 1991, "Mapping models for Hamiltonian systems with applications to resonant asteroid motion". In Predictability, Stability and Chaos in N-Body Dynamical Systems (A.E.Roy, ed.), Plenum Press, New York, 157-175.

Henrard, J., Watanabe, N. and Moons, M.: 1995, "A bridge between secondary resonances and secular resonances inside the Hecuba gap". Icarus 115, 336-346.

Morbidelli, A. and Moons, M.: 1993, "Secular resonances in the mean motion commensurabilities: The $2 / 1$ and $3 / 2$ cases". Icarus 102, 316-332.

Nobili, A.M., Milani, A. and Carpino, M.: 1989, "Fundamental frequencies and small divisors in the orbits of the outer planets". Astron. Astrophys. 210, 313-336.

Simon, J.L. and Bretagnon.P.: 1975, "Results of first-order perturbations of the four large planets" Astron. Astrophys. Suppl. 22, 107-160. 